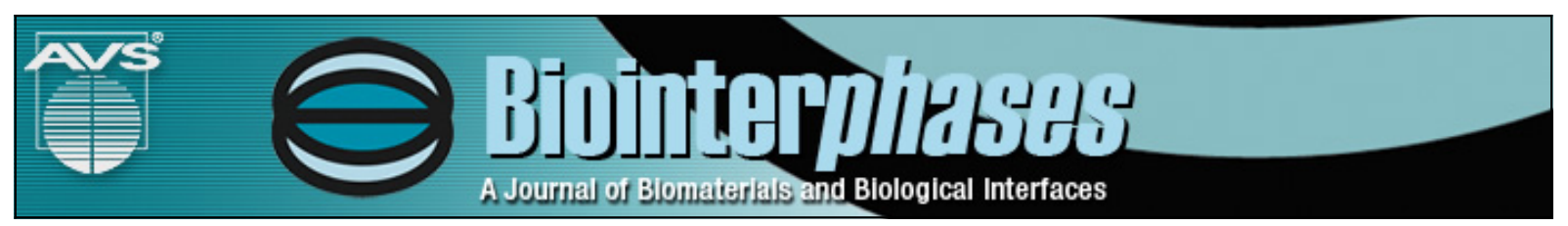

Adhesion kinetics of human primary monocytes, dendritic cells, and macrophages:

Dynamic cell adhesion measurements with a label-free optical biosensor and their comparison with end-point assays

Norbert Orgovan, Rita Ungai-Salánki, Szilvia Lukácsi, Noémi Sándor, Zsuzsa Bajtay, Anna Erdei, Bálint Szabó, and Robert Horvath

Citation: Biointerphases 11, 031001 (2016); doi: 10.1116/1.4954789

View online: http://dx.doi.org/10.1116/1.4954789

View Table of Contents: http://scitation.aip.org/content/avs/journal/bip/11/3?ver=pdfcov

Published by the AVS: Science \& Technology of Materials, Interfaces, and Processing

Articles you may be interested in

Quick chip assay using locked nucleic acid modified epithelial cell adhesion molecule and nucleolin aptamers for the capture of circulating tumor cells

Biomicrofluidics 9, 054110 (2015); 10.1063/1.4930983

Interaction between drug delivery vehicles and cells under the effect of shear stress

Biomicrofluidics 9, 052605 (2015); 10.1063/1.4923324

Regulation of cell migration and osteogenic differentiation in mesenchymal stem cells under extremely low fluidic shear stress

Biomicrofluidics 8, 052008 (2014); 10.1063/1.4896557

A microfluidic model for organ-specific extravasation of circulating tumor cells

Biomicrofluidics 8, 024103 (2014); 10.1063/1.4868301

Thiolene-based microfluidic flow cells for surface plasmon resonance imaging

Biomicrofluidics 5, 026501 (2011); 10.1063/1.3596395 


\title{
Adhesion kinetics of human primary monocytes, dendritic cells, and macrophages: Dynamic cell adhesion measurements with a label-free optical biosensor and their comparison with end-point assays
}

\author{
Norbert Orgovan ${ }^{\mathrm{a})}$ \\ Department of Biological Physics, Eötvös University, Pázmány P. stny. 1/A, H-1117 Budapest, Hungary \\ and Nanobiosensorics Group, Hungarian Academy of Sciences, Centre for Energy Research, Institute \\ for Technical Physics and Materials Science, Konkoly-Thege út 29-33, H-1120 Budapest, Hungary
}

Rita Ungai-Salánki

Nanobiosensorics Group, Hungarian Academy of Sciences, Centre for Energy Research, Institute

for Technical Physics and Materials Science, Konkoly-Thege út 29-33, H-1120 Budapest, Hungary

and Doctoral School of Molecular-and Nanotechnologies, University of Pannonia, H-8200 Veszprém, Hungary

Szilvia Lukácsi

Department of Immunology, Eötvös University, Pázmány P. stny. 1/C, H-1117 Budapest, Hungary

Noémi Sándor

MTA-ELTE Immunology Research Group, ELTE, Pázmány P. stny. 1/C, H-1117 Budapest, Hungary

Zsuzsa Bajtay

Department of Immunology, Eötvös University, Pázmány P. stny. 1/C, H-1117 Budapest, Hungary

Anna Erdei

Department of Immunology, Eötvös University, Pázmány P. stny. 1/C, H-1117 Budapest, Hungary and MTA-ELTE Immunology Research Group, ELTE, Pázmány P. stny. 1/C, H-1117 Budapest, Hungary

\section{Bálint Szabó}

Department of Biological Physics, Eötvös University, Pázmány P. stny. 1/A, H-1117 Budapest, Hungary and Nanobiosensorics Group, Hungarian Academy of Sciences, Centre for Energy Research, Institute for

Technical Physics and Materials Science, Konkoly-Thege út 29-33, H-1120 Budapest, Hungary

\section{Robert Horvath}

Nanobiosensorics Group, Hungarian Academy of Sciences, Centre for Energy Research, Institute for Technical Physics and Materials Science, Konkoly-Thege út 29-33, H-1120 Budapest, Hungary

(Received 17 February 2016; accepted 14 June 2016; published 1 July 2016)

\begin{abstract}
Monocytes, dendritic cells (DCs), and macrophages (MFs) are closely related immune cells that differ in their main functions. These specific functions are, to a considerable degree, determined by the differences in the adhesion behavior of the cells. To study the inherently and essentially dynamic aspects of the adhesion of monocytes, DCs, and MFs, dynamic cell adhesion assays were performed with a high-throughput label-free optical biosensor [Epic BenchTop (BT)] on surfaces coated with either fibrinogen (Fgn) or the biomimetic copolymer PLL- $g$-PEG-RGD. Cell adhesion profiles typically reached their maximum at $\sim 60 \mathrm{~min}$ after cell seeding, which was followed by a monotonic signal decrease, indicating gradually weakening cell adhesion. According to the biosensor response, cell types could be ordered by increasing adherence as monocytes, MFs, and DCs. Notably, all three cell types induced a larger biosensor signal on Fgn than on PLL-g-PEG-RGD. To interpret this result, the molecular layers were characterized by further exploiting the potentials of the biosensor: by measuring the adsorption signal induced during the surface coating procedure, the authors could estimate the surface density of adsorbed molecules and, thus, the number of binding sites potentially presented for the adhesion receptors. Surfaces coated with PLL-g-PEG-RGD presented less RGD sites, but was less efficient in promoting cell spreading than those coated with Fgn; hence, other binding sites in Fgn played a more decisive role in determining cell adherence. To support the cell adhesion data obtained with the biosensor, cell adherence on Fgn-coated surfaces 30-60 min after cell seeding was measured with three complementary techniques, i.e., with (1) a fluorescence-based classical adherence assay, (2) a shear flow chamber applying hydrodynamic shear stress to wash cells away, and (3) an automated micropipette using vacuum-generated fluid flow to lift cells up. These techniques confirmed the results obtained with the high-temporal-resolution Epic BT, but could only provide end-point data. In contrast, complex, nonmonotonic cell adhesion kinetics measured by the high-throughput optical biosensor is expected to open a window on the hidden background of the immune cell-extracellular matrix interactions. (C) 2016 American Vacuum Society. [http://dx.doi.org/10.1116/1.4954789]
\end{abstract}

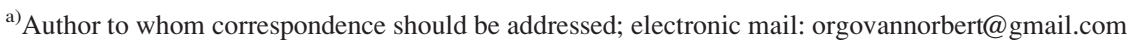




\section{INTRODUCTION}

Monocytes (a type of white blood cells, leukocytes) developed in the bone marrow are pivotal cellular elements of the innate immune system that can initiate and regulate immune responses. ${ }^{1}$ After an initial period of few days monitoring foreign substances in the blood stream, monocytes migrate through the endothelium of the blood vessels to the neighboring tissues and differentiate into more specialized dendritic cells (DCs) or macrophages (MFs). ${ }^{2}$ During their life cycle, these cells often get into contact with the extracellular matrix (ECM), i.e., thick layers of highly diverse interconnected macromolecules. The ECM functions not only as a mechanical cell scaffold but also as a signaling platform ${ }^{3}$ which modulates the inflammatory immune response ${ }^{4-7}$ by providing various physical and biochemical clues to immune cells encountering them. For instance, fibrinogen (Fgn), ${ }^{8}$ an ECM protein and a ligand for a number of adhesion receptors, often accumulates at inflammation sites and, therefore, helps immune cells to find places of injury. ${ }^{9}$ Moreover, chronic inflammation may lead to the degradation of the ECM via inflammatory cytokines and proteases, generating bioactive fragments (for example, RGD-containing peptides) which influence the activity and function of infiltrating and resident cells. ${ }^{10}$

All the fundamental interactions between immune cells and their surroundings (e.g., other self-cells of an organism, pathogens, or the biopolymers of the ECM) are predominantly mediated by a certain set of adhesion receptors expressed by the cells. ${ }^{11,12} \alpha \beta$ heterodimer transmembrane protein integrins - which are the major receptors anchoring the cells to the ECM and establishing a connection between the latter and the cytoskeleton-are particularly deeply involved in integrating and transducing ECM-provided signals. ${ }^{13}$ Furthermore, integrins also function as bidirectional signaling machines, ${ }^{14}$ and thus, they tightly control leukocyte migration to the target tissue, ${ }^{15,16}$ gene expression, ${ }^{17}$ cell proliferation, differentiation, ${ }^{18,19}$ and more. The most abundant integrins expressed by monocytes and monocytederived cells are leukocyte-specific $\beta 2$-integrins, ${ }^{20,21}$ especially $\alpha_{\mathrm{M}} \beta_{2}$ (also termed as complement receptor 3, Mac-1 or $\mathrm{CD} 11 \mathrm{~b} / \mathrm{CD} 18$ ) and $\alpha_{\mathrm{X}} \beta_{2}$ (also termed as complement receptor 4 or $\mathrm{CD} 11 \mathrm{c} / \mathrm{CD} 18)$. It is long known that the differentiation of monocytes into macrophages and to dendritic cells is accompanied by alterations in the expression levels of these integrins. ${ }^{22,23}$ In the case of immune cells, which may have various contact partners other than the ECM or other self-cells, integrin expression levels shape cell-typespecific functions to a considerable degree. Here, we aimed at shedding light on the differences in the time-dependent adhesive behavior of monocytes, DCs, and MFs.

In the past decades, the field has attracted an increasingly broad scientific interest, and the interaction of either monocytes, MFs, or DCs with various proteins (including fibrinogen, ${ }^{9,24}$ collagen-I, ${ }^{25}$ laminin, ${ }^{26}$ serum proteins, ${ }^{27,28}$ and biomimetic peptides based on functional sites of fibronec$\operatorname{tin}^{29,30}$ ) have been investigated. However, lacking the appropriate technique, the overwhelming majority of previous studies failed to capture the inherent and essential dynamics of immune cell adhesion, and characterized it only at a single time point.

When it comes to measuring the kinetics of cell adhesion, nor the techniques based on direct measurements of the adhesion force (e.g., variants of atomic force microscopy, ${ }^{31,32}$ automated micropipette, ${ }^{24}$ and traction force microscopy ${ }^{33,34}$ ), neither optical microscopy for the visualization of cells or their adhesion-associated molecular structures (e.g., wide-field, confocal, total internal reflection microscopies $^{35}$ ) can compete with cell-based label-free biosensors which enable the high temporal-resolution monitoring of the activity of surface-adhered cells. Evanescent field-based optical biosensors, including surface plasmon resonance (SPR), ${ }^{36,37}$ optical waveguide lightmode spectroscopy (OWLS), ${ }^{28,38-40}$ photonic crystal biosensors, ${ }^{41}$ grating coupling interferometry, ${ }^{42,43}$ and resonant waveguide grating (commonly recognized as Epic) ${ }^{44,45}$ biosensors, are considered to be especially straightforward means to monitor surface adhesion. First, they are sensitive only in a $\sim 150 \mathrm{~nm}$ thick layer closest to the substratum, exactly where establishment and maturation of anchorage and spreading occurs. Second, they are closer to a hypothetical zero-perturbation system than other adhesion-measuring techniques in- or outside the family of label-free biosensors. Third, their response integrating changes in both the size of the contact area and the optical density therein (dependent on the extents of actin polymerization, integrin clustering, adhesion complex formation, and maturation) is proportional to the strength of adhesion. ${ }^{40}$ Nonetheless, the permeation of such biosensors to adhesion science has long been delayed partly due to the lack of high throughput in first-generation devices (OWLS and SPR), which is practically a requirement in cell research. High throughput is increasingly critical for studying primary immune cells, e.g., since they are particularly sensitive and responsive to changes in their environment, and since monocytes can undergo spontaneous differentiation.

Here, we utilized a high-throughput label-free optical biosensor to monitor cell adhesion and spreading in real time [Fig. 1(a)]. In our former study, we demonstrated the potentials of a first-generation optical biosensor, namely, the OWLS, to measure the adhesion kinetics of primary monocytes in the presence of varying serum concentrations. ${ }^{28}$ However, single-channel OWLS lacked the throughput which was necessary to simultaneously measure the adhesion of different types of cells on various surfaces. Thus, here we used the Epic BenchTop (BT), a microplate-based device $^{45}$ relying on the same detection principle as OWLS, to study the adhesion kinetics of monocytes isolated from human blood and that of in vitro differentiated, monocytederived macrophages (MDMs), and monocyte-derived dendritic cells (MDCs). Specific adhesion was investigated on substrata coated with either fibrinogen, or a biomimetic copolymer, PLL- $g$-PEG-RGD.

To support the biosensor data, cell adherence 30-60 min after cell seeding was further tested with three complementary 

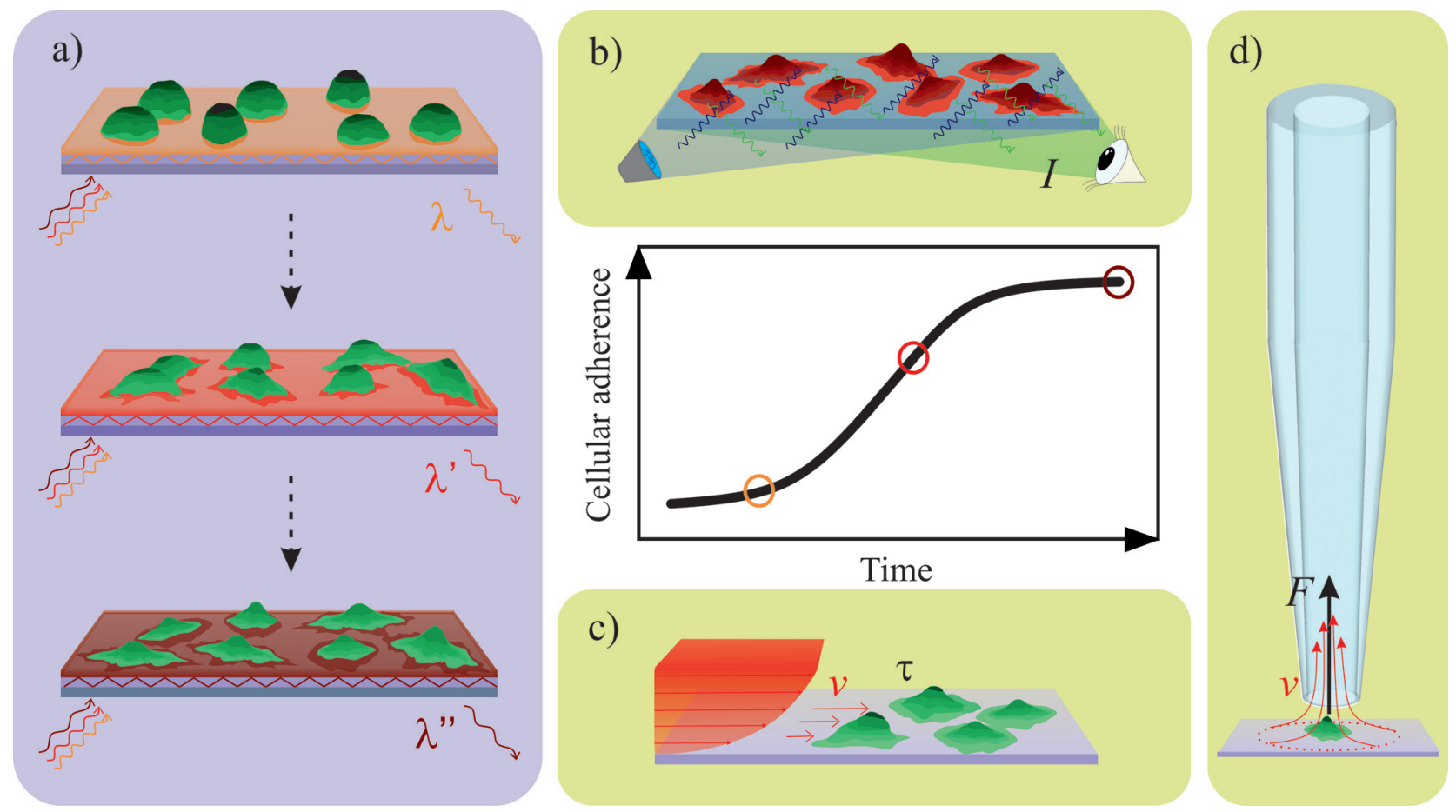

FIG. 1. Schematic illustration of the working principle of the techniques employed to characterize immune cell adherence (not to scale). Panel (a): the detection principle of the high-throughput optical biosensor. The wavelength-tuned illuminating light (illustrated as ingoing orange/red/plum waved arrows) is incoupled into the planar waveguides (i.e., sensing units) of the biosensor microplate. The illuminating light penetrates in a $\sim 150$ nm thick layer closest the sensor surface, and thus probes the local refractive index. However, at given refractive index relations, only a single wavelength is able to propagate in the waveguide layer (such guided light is illustrated as an orange/red/plum zigzag line in the different cases). During cell spreading, an increasingly bigger portion of the cell body enters into the layer probed by the illuminating light (from top to bottom illustrated as increasingly bigger contact areas colored in orange/red/plum), altering the local refractive index. As a consequence, waveguiding is ceased at the original wavelength $\lambda$ (illustrated in orange, at the top), but resumes at wavelength $\lambda^{\prime} \neq \lambda$ (illustrated in red, in the middle), then at $\lambda^{\prime \prime} \neq \lambda^{\prime} \neq \lambda$ (illustrated in plum, at the bottom). After a few micrometers of propagation, light is outcoupled from the waveguide (illustrated as outgoing waved arrows), so its wavelength can be detected. Thus, cell spreading can be monitored in a labelfree manner as a change in the wavelength of the guided light $(\Delta \lambda)$. Panel $(b)$ : adherence measurement in a classical end-point assay. Cells are labeled with a fluorescent dye (illustrated as cells colored in red instead of green), then seeded and incubated on the surface of interest. After incubation, unattached cells are removed via gentle washing, and the number of attached cells is determined by comparing their fluorescent intensity $(I)$ to that measured on a reference surface which was not washed after incubation (exciting and fluorescent lights are illustrated in blue and green, respectively). Panel (c): adherence measurement in a flow chamber (rectangular microfluidic channel). Cells introduced into the flow chamber are allowed to adhere to its bottom. After incubation, the flow chamber is placed on a microscope to visualize the cells, then a laminar fluid flow [resulting in a parabolic flow velocity profile $v(\mathrm{z})$ exhibiting a shear stress $\tau$ on cells] is applied for a couple of seconds. Flow rate is increased in several steps until most cells are removed. Adherence is characterized as the ratio of adherent cells at a given flow rate. Panel (d): adherence measurement with the automated micropipette. The computer-controlled micropipette uses a vacuum-generated fluid flow (with flow rate $v$, streamlines illustrated as red arrows) to test the adherence of single cells. Estimated lifting force $(F$, black arrow) acting on cells can be determined with computational methods. Adherence is characterized as the ratio of adherent cells at a given lifting force. Panel in the middle: temporal evolution of cellular adherence is usually well described with a sigmoid function. Importantly, among the presented techniques, only the label-free biosensor enables the kinetics of cell adhesion to be monitored [illustrated for three time points in Fig. 2(a)].

techniques, i.e., with (1) a classical, static adherence assay, (2) a flow chamber-based assay, and (3) an automated micropipettebased assay [Figs. 1(b)-1(d)].

\section{MATERIALS AND METHODS}

\section{A. Monocyte isolation from human blood}

Buffy coat was obtained from healthy donors and provided by the Hungarian National Blood Transfusion Service. All experiment protocol was approved by the "Committee of Science and Research Ethics" of the "Medical Research Council" of Hungary ("ETT TUKEB"). The methods were carried out in accordance with the approved guidelines of the Declaration of Helsinki.

Peripheral blood mononuclear cells were isolated from buffy coat by density gradient centrifugation on Ficoll-Paque (GE Healthcare). Unlabeled monocytes were isolated by negative magnetic separation using the Miltenyi Monocyte Isolation kit II (Miltenyi) according to the manufacturer's instructions. Briefly, nonmonocytes were indirectly magnetically labeled using a cocktail of biotin-conjugated antibodies and antibiotin MicroBeads. Highly enriched unlabeled monocytes were obtained by depletion of the magnetically labeled cells. Cells were cultivated in the CellGro GMP DC (CellGenix GmbH, Freiburg, Germany) serum-free medium at $37^{\circ} \mathrm{C}, 5 \% \mathrm{CO}_{2}$ atmosphere. To avoid spontaneous cell attachment to the dish, cells were cultured in Teflon-coated flasks.

\section{B. Differentiation of monocytes into dendritic cells and macrophages}

To generate MDCs in vitro, isolated monocytes were cultivated in CellGro serum-free DC medium (CellGenix 
$\mathrm{GmbH}$ ) supplemented with $100 \mathrm{ng} / \mathrm{ml}$ recombinant human granulocyte/macrophage-colony-stimulating factor (GMCSF, R\&D systems) and $15 \mathrm{ng} / \mathrm{ml}$ recombinant human interleukin-4 (R\&D systems) for 5 days in 24-well cell culture plates (Corning) at a cell density of $5 \times 10^{5} / \mathrm{ml}$. To generate MDMs, cells were cultivated as MDCs except that only GM-CSF cytokine was added to the culture. Cytokines were supplemented every 3 days.

To identify MDCs and MDMs, cultures were checked with flow cytometry based on the fact that MDCs are CD14-, while MDMs are CD14+ cells. Furthermore, we analyzed the cell morphology in the cultures by inverted microscope. MDCs were floating at day 5 of differentiation and had several visible dendrites. Macrophages were more attached to the culture plate, had a more rounded shape as compared to MDCs, and had no dendrites.

For the adhesion experiments, cells were harvested by pipetting after vigorously suspending the cultures. MDMs only loosely adhered to the bottom of the culture dish, while MDCs did not adhere to it at all; thereby, no scraping was needed to detach the cells. Monocytes were used immediately after separation.

\section{Coating solution preparation and surface coating procedure}

The synthetic copolymers, poly(L-lysine)-graft-poly(ethylene glycol) (PLL-g-PEG), and its RGD-functionalized counterpart, PLL- $g$-PEG/PEG-GGGGYGRGDSPG (simply referred to as PLL- $g$-PEG-RGD throughout this work), were obtained as powders (SuSoS AG). The materials were stored at $-20^{\circ} \mathrm{C}$ until use. Each powder was then dissolved in $10 \mathrm{mM} 4-(2-$ hydroxyethyl)-1-piperazine ethanesulfonic acid (HEPES, from Sigma-Aldrich Chemie $\mathrm{GmbH}$ ) at $\mathrm{pH} 7.4$ to obtain coating solutions with a concentration of $250 \mu \mathrm{g} / \mathrm{ml}$. These were then sterile-filtered and stored at $4{ }^{\circ} \mathrm{C}$ for a maximum of 2 weeks.

Fgn (340 kDa, from Merck KGaA) was dissolved in ultrapure $\mathrm{H}_{2} \mathrm{O}$ and stored as $36 \mathrm{mg} / \mathrm{ml}$ stock solutions at $-80^{\circ} \mathrm{C}$ until use. After gentle thawing, this was diluted in phosphate buffered salt solution (PBS, Sigma-Aldrich) to a final concentration of $10 \mu \mathrm{g} / \mathrm{ml}$.

Surface coatings were created by physisorption from the appropriate coating solutions. First, designated surfaces were treated with a $10 \mu \mathrm{g} / \mathrm{ml}$ Fgn coating solution, and incubated for $1 \mathrm{~h}$ at $37^{\circ} \mathrm{C}$ (except for the case of the biosensor, where this step was preceded by a baseline read with PBS in the wells). The Fgn solution was then removed, and surfaces were rinsed three times with PBS. Next, unoccupied surface areas on Fgn-coated surfaces were passivated with PLL-gPEG, and surfaces not treated yet were coated with either PLL- $g$-PEG for negative control, or with PLL- $g$-PEG-RGD $(250 \mu \mathrm{g} / \mathrm{ml}, 30 \mathrm{~min}$, room temperature). These solutions were then removed, and surfaces were rinsed three times with PBS.

The proteins and the peptide ligate used in the experiments were not of bacterial source; therefore, the probability of their lipopolysaccharide (LPS) contamination was very low-which is a crucial question, since monocytes, MDMs, and MDCs all express TLR4, and hence, they are susceptible to LPS. Nonetheless, we tested whether the components used for the assays induced the activation of the cells by measuring their tumor necrosis factor alpha (TNF- $\alpha$, an inflammatory cytokine) production with an enzmye-linked immunosorbent assay (TNF- $\alpha$ DuoSet ELISA was obtained from R\&D). We found that neither of the employed substances induced TNF- $\alpha$ production of monoctyes, MDMs, or MDCs. Moreover, we tested the different coatings using a modified U937 monocytoid cell line that produces green fluorescent protein (GFP) when activated with the protein nuclear factor kappa-lightchain-enhancer of activated B cells $(\mathrm{NF}-\kappa \mathrm{B}) .{ }^{46}$ Activation of these cells was measured with fluorescent microscopy, and these experiments further confirmed that no NF- $\kappa \mathrm{B}$ activation occurred under the used experimental conditions.

\section{High-throughput label-free detection with the Epic BT}

Epic BT is a next-generation biosensor enabling highthroughput label-free detection at a solid-liquid interface [Fig. 1(a)]. Epic BT accepts 96- or 384-well SBS-format Epic microplates. The bottom of each microplate serves as an optical waveguide, in which the illuminating light can be coupled via optical gratings integrated at the central positions of the wells. Light beams in the waveguide interfere with each other; destructive interference precludes waveguiding, while constructive interference leads to resonance and to the excitation of a guided lightmode. The latter can be achieved only at a discrete illuminating wavelength, called resonant wavelength $(\lambda)$. The guided lightmode generates an exponentially decaying evanescent field in a 100-200 nm width layer over the sensor, which probes refractive index (RI) variations at this interface. $^{40,45}$ Any process accompanied by RI-variations in this layer (bulk RI change, molecular adsorption, cell spreading, or dynamic redistribution inside the attached cells) untunes the resonance by altering the phase-shift of the propagating light when it is reflected from the interface. This leads to destructive interference at the original wavelength and to a constructive interference at a slightly different wavelength, i.e., waveguiding resumes at an illuminating wavelength $\lambda^{\prime} \neq \lambda$. The primary output of the Epic BT is the shift of the resonant wavelength, $\Delta \lambda=\lambda^{\prime}-\lambda$. This is monitored in real time, enabling kinetic measurements. The biosensor response is directly proportional to the strength of adhesion. ${ }^{40}$

In practice, Epic BT uses a broadband light source and a tunable optical filter to simultaneously interrogate all wells of the microplate every $3 \mathrm{~s}$ by sweeping the wavelength from 823 to $838 \mathrm{~nm}$ with a $0.25 \mathrm{pm}$ wavelength resolution. The guided wavelength is outcoupled by the same grating used for incoupling, and the resonant wavelength distribution within each well is imaged with a spatial resolution of $\sim 90 \mu \mathrm{m}$ using a CCD camera. The created two-dimensional resonant wavelength map allows patterns in single wells (corresponding to areas of, e.g., aggregated spread cells, or 
dead ones) to be identified and permits data filtration to improve assay quality. ${ }^{47}$

\section{E. Dynamic cell adhesion assay on the Epic BenchTop system}

The assay medium for cell spreading experiments was prepared by supplementing the CellGro GMP DC (CellGenix $\mathrm{GmbH}$ ) serum-free medium with $20 \mathrm{mM}$ HEPES (from Sigma-Aldrich Chemie $\mathrm{GmbH}), 0.25 \mu \mathrm{g} / \mathrm{ml}$ amphotericin, and $40 \mu \mathrm{g} / \mathrm{ml}$ gentamycin.

First, a baseline was established with the assay medium in the wells. Meanwhile, cells were centrifuged at $380 \mathrm{~g}$ for $5 \mathrm{~min}$, and the cell pellet was resuspended in fresh assay medium with gentle pipetting. Cells were then counted in a hemocytometer, and $\sim 8000$ cells in a volume of $\sim 50 \mu \mathrm{l}$ were added to the sensor wells already containing $20 \mu \mathrm{l}$ assay medium. All experiments were done in triplicates in three different wells at room temperature. Averaging every five subsequent data points, the effective sampling rate was $1 / 15 \mathrm{~s}^{-1}$.

In all the experiments carried out with the biosensor, 384well uncoated Epic microplates (Corning) were used. Wells unused in an experiment were covered with a special aluminum foil (Corning) to prevent their contamination. Except for the first step of the surface coating procedure, all steps of the biosensor experiments were conducted at room temperature.

\section{F. CFDA-SE labeling of cells}

Directly before the classical adherence measurement (see Sec. II G), cells at a density of $10^{6}$ cells $/ \mathrm{ml}$ were freshly labeled with $10 \mu \mathrm{M}$ carboxyfluorescein diacetate succinimidyl ester (CFDA-SE, from Molecular Probes) fluorescent dye. Briefly, cells were mixed with appropriate dye concentration and incubated for $10 \mathrm{~min}$ at room temperature in dark. Then, they were washed three times with phosphate buffered saline (PBS) containing 5\% fetal calf serum (FCS) to remove excess dye.

\section{G. Classical end-point adhesion assay}

In classical end-point adherence assays, the ratio of adherent cells was accessed by measuring the fluorescent intensity of adherent populations relative to that of an appropriate control [Fig. 2(b)].

Wells of a 96-well polystyrene plate (Greiner) were coated according to the protocol described in Sec. II. Subsequently, wells were washed two times with PBS, and 500000 CFDA-SE-labeled cells (for the labeling protocol, see Sec. IIF) suspended in $100 \mu$ l Rosewell Park Memorial Institute medium (RPMI, obtained from Sigma-Aldrich) medium supplemented with 10\% FCS (Gibco) were pipetted into each well. Cells were allowed to adhere for $1 \mathrm{~h}$ at $37^{\circ} \mathrm{C}$, $5 \% \mathrm{CO}_{2}$ atmosphere. Afterward, fluorescence intensity was measured with a Thermo Fluoroskan Ascent microplate reader (Thermo Scientific) to determine the maximal fluorescence in each well. Then, wells were washed with warm RPMI-10\% FCS two times to remove all unbound cells.
Finally, wells were loaded with $100 \mu$ l RPMI-10\% FCS, and fluorescence intensity was measured again to determine the fluorescence of adhered cells. The amount of adhered cells was determined by comparing the fluorescence intensity in each well before and after washing.

\section{H. Flow chamber-based adhesion assay}

In flow-chamber based assays, well-defined shear stresses were applied to test the adherence of a population of cells [Fig. 2(c)], which was then characterized by the ratio of the adherent cells to the total number of cells initially seeded $\left(2-4 \times 10^{6}\right.$ cells $\left./ \mathrm{ml}\right)$.

We used six-channel plastic flow chambers (Ibidi, $\mu$ Slide VI 0.1) in the experiments. The channels were coated with Fgn and/or passivated with PLL- $g$-PEG as detailed in Sec. II. Then, cells suspended in RPMI-10\% FCS were introduced into the precoated channels and incubated for $30 \mathrm{~min}$ at $37^{\circ} \mathrm{C}$ in $5 \% \mathrm{CO}_{2}$ atmosphere. After incubation, the flow chamber was placed on an inverted phase contrast microscope (Olympus CKX41, $4 \times$ objective lens) to monitor the detachment of cells in the flow. A $50 \mathrm{ml}$ syringe controlled by a syringe pump (New Era NE-1000) was filled with Hank's buffered salt solution, and it was connected to the $\mu$-Slide channel via polytetrafluoroethylene tubing. The flow rate was increased in several steps as long as most of the cells were removed from the coated surface. Flow was applied for $10 \mathrm{~s}$ at each step. Images were captured to determine the number of cells remaining on the surface before initiating the flow and after each step.

\section{Automated micropipette-based adhesion assay}

The recently introduced automated micropipette utilizes a precisely controlled, vacuum-generated fluid flow to test the adherence of single cells [Fig. 2(d)]. ${ }^{24}$ Hydrodynamic lifting force acting on cells can be estimated from the experimentally set vacuum values by simulating the fluid flow in the micropipette. ${ }^{24}$ Increasing the vacuum in several steps, cellular adherence is characterized by the ratio of still adherent cells to the total number of cells initially seeded. (Individual cells picked up by the micropipette can be automatically deposited in submicroliter drops for further analysis, see Ref. 48.)

A constrained region of a $35 \mathrm{~mm}$ plastic tissue culture petri dish (Greiner) was coated with Fgn, ${ }^{24}$ then the entire bottom of the Petri dish was treated with PLL- $g$-PEG according to the coating protocol described in Sec. II. Next, 75000 cells suspended in RPMI-10\% FCS were seeded into the dish, and incubated for $30 \mathrm{~min}$ at $37^{\circ} \mathrm{C}$ in $5 \% \mathrm{CO}_{2}$ atmosphere. Subsequently, floating cells were gently washed away, and cells attached to the surface were scanned on a motorized inverted microscope and recognized by computer vision. A glass micropipette with an inner diameter of $70 \mu \mathrm{m}$ attached to a vertically motorized micromanipulator was maneuvered to each detected cell one by one automatically. Cell adhesion was probed by applying a precisely controlled fluid flow through the micropipette. The adhesion force of 

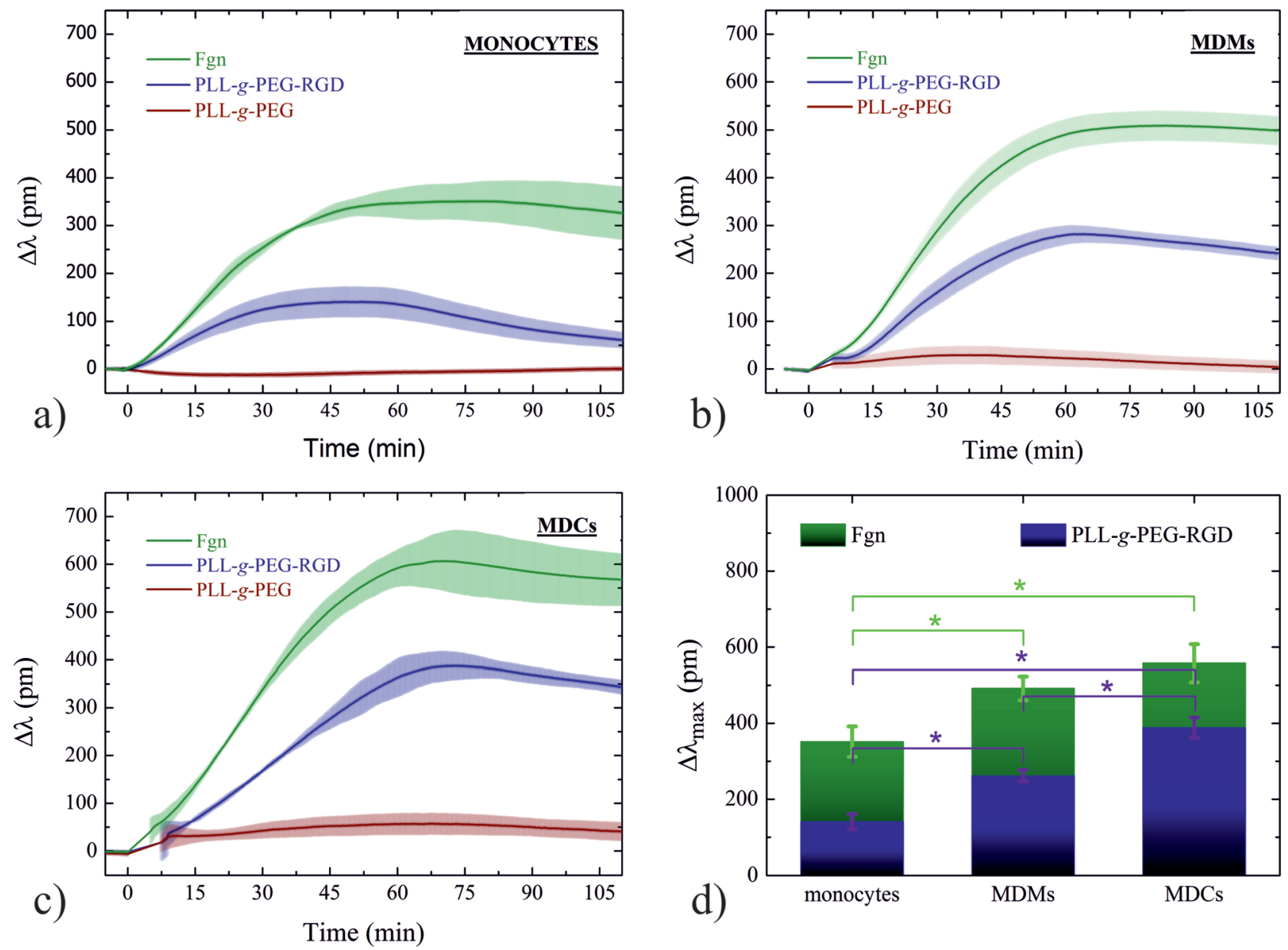

Fig. 2. Time-dependent adherence of monocytes, MDMs, and MDCs on PLL- $g$-PEG- and Fgn-coated surfaces, as was measured with the Epic BT in DCA assays. Representative kinetic profiles provoked by adhering and spreading monocytes, MDMs, and MDCs are shown in panels (a)-(c), respectively. PLL- $g$-PEG-coated surfaces were used as negative control. The background-corrected maximum biosensor signals induced by different cells on PLL- $g$-PEGRGD- and Fgn-coated surfaces are shown in panel (d). In all panels data are shown as means, error bars represent standard deviations. * indicates statistical significance with $p<0.05$ (t-test).

cells could be accurately measured by repeating the pick-up process with increasing vacuum.

\section{J. Statistical analysis}

All adhesion data shown in the figures were analyzed with two-sample unpaired (one-tailed) t-tests. Differences were considered statistically significant if $p<0.05$.

\section{RESULTS AND DISCUSSION}

Here, we used a microplate-based high-throughput optical biosensor, the Epic BT (see Fig. 1 and Sec. II), to study the adhesion kinetics of monocytes isolated from human blood and that of in vitro differentiated MDMs, and MDCs (for the isolation and differentiation protocols, see Secs. II A and II B). Specific adhesion was investigated on substrata coated with either fibrinogen, or a biomimetic copolymer, PLL$g$-PEG-RGD (for the coating protocols, see Sec. II C).

The high-throughput optical biosensor [Fig. 1(a)] enabled the kinetics of the adhesion and spreading of a cell population to be monitored with high temporal resolution and without the need to incorporate labels that could potentially disturb cell behavior.

\section{A. Leukocyte adhesion often exhibit nontrivial kinetics}

Spreading kinetics obtained by monitoring the activity of different cell types seeded onto different substrata are shown in Fig. 2. First, note that nonspecific adhesion could be effectively blocked by precoating the sensor surface with the biologically inactive PLL- $g$-PEG. In contrast, surfaces coated with either Fgn or PLL-g-PEG-RGD induced specific cell adhesion. The kinetics of spreading was similar for all three cell types; a typical adhesion kinetic profile obtained its maximum around $60 \mathrm{~min}$ after cell seeding, then the signal started to slowly decrease. This latter characteristic was unexpected, as normally (i.e., in the absence of treatment, stimulation, perturbation) the spreading curve of most cell types resembles a symmetrical sigmoid with a stabile plateau at equilibrium. ${ }^{40,45,49,50}$ The extent of signal decrease relative to the maximum biosensor signal showed strong dependence on donors, as well as on cell types. The signal produced by monocytes and MDCs exhibited more prominent relative 
decrease than that given by MDMs. Signal decrease was observed not only when the cells were cultured and assayed in serum-free medium, but also when kept in $10 \%$ FCSRPMI (data not shown). The nontrivial kinetics implies complex adhesive behavior, probably with multiple cellular activities acting in concert and having partially opposing effects on total cell adherence and spreading. For instance, podosomes - the multifunctional adhesive and invasive structures that are particularly prominent in the monocytic lineage - are deeply engaged in both cell-substrate adhesion and matrix degradation. ${ }^{51}$ Thus, we can speculate that within the first hour after cell seeding the biosensor signal was dominated by the spreading process, but then another effect turned on. This could be related to the transition of the cell shape from rounded to more complex shapes with long protrusions suggested by the imaging of cells during spreading (data not shown).

\section{B. Monocytes, macrophages, and dendritic cells differ in their cell adherence}

As expected from differences in cell size (the diameter of unspread monocytes are approximately half the diameter of MDCs and MDMs) and in adhesion receptor expression levels, ${ }^{22}$ differentiated cells induced larger biosensor signals (indicating firmer adherence) than monocytes. Notably, these cell-type-related differences in cell adherence were more pronounced on PLL- $g$-PEG-RGD, than on Fgn-coated surfaces (Fig. 2).

Figure 2 also indicates that the maximum biosensor signal given by MDCs was larger both on Fgn- and PLL- $g$-PEGRGD-coated surfaces than that provoked by MDMs. This result is in accordance with previously published data. First, the most abundant integrins on the surface of both cell types are leukocyte-specific integrins (especially, $\alpha_{\mathrm{M}} \beta_{2}$ and $\alpha_{\mathrm{X}} \beta_{2}$ heterodimers, among which $\alpha_{\mathrm{M}} \beta_{2}$ dominates), and it seems that MDCs have higher overall expression levels of such adhesion receptors than MDMs. ${ }^{22}$ More recently, we tested the adherence of MDCs and MDMs to Fgn with a computer controlled micropipette, and found that a larger sucking force was necessary to remove MDCs from the surface than to remove MDMs. ${ }^{24}$

\section{Fibrinogen-coated surfaces promote cell adhesion more effectively than PLL-g-PEG-RGD layers, although the latter presents more RGD motifs}

All three cell types induced a markedly larger biosensor signal on Fgn-coated surfaces than on PLL-g-PEG-RGD (Fig. 2). To interpret this result, we characterized both surfaces by further exploiting the potentials of the biosensor. By measuring the biosensor signals induced during the surface coating procedure, one can estimate the surface density of adsorbed molecules and, thus, the number of binding sites potentially presented for the adhesion receptors.

During adsorption, both PLL-g-PEG-RGD and Fgn induced a wavelength shift of $\sim 420 \mathrm{pm}$ (data not shown). Previously, we have used the dual-mode OWLS, enabling the adsorbed surface mass density $(M)$ to be determined, as a complementary technique to calibrate the surface sensitivity of the Epic BT. We have established the following relation: ${ }^{52}$

$$
\Delta M \approx \Delta \lambda \times 3.1 \times 10^{-1} \mathrm{ng} / \mathrm{cm}^{2} \mathrm{pm}
$$

Hence, the measured $\sim 420$ pm wavelength shift corresponds to a surface mass density of $\sim 130 \mathrm{ng} / \mathrm{cm}^{2}$. The number of molecules on the surfaces can be straightforwardly determined by using this latter data and the molecular weights of PLL-g-PEG-RGD (77.1 kDa) and Fgn (340 kDa). To calculate the number of binding sites potentially presented to cells, however, the number of binding sites per molecule has to be also known. Due to its design, surface-absorbed PLL- $g$-PEG-RGD has a single sequence that cells can interact with; the RGD-motif (an average of 3.1 RGDs are present per molecule). This tripeptide is recognized by a specific subgroup of integrins, among which leukocytes express $\alpha_{5} \beta_{1}$ and $\alpha_{5} \beta_{3}{ }^{53}$ However, the most abundant integrins expressed by leukocytes $\left(\alpha_{\mathrm{X}} \beta_{2}\right.$ and $\left.\alpha_{\mathrm{M}} \beta_{2}\right)$ are not involved in RGD-binding. (Albeit in whole cell experiments RGDcontaining peptides could inhibit $\alpha_{\mathrm{M}} \beta_{2}$ function, later it has been shown that this was not due to a direct interaction between $\alpha_{M} \beta_{2}$ and RGD, but rather due to crosstalk between $\alpha_{\mathrm{M}} \beta_{2}$ and receptors that do actually recognize RGD. ${ }^{54}$ ) In contrast, fibrinogen, a key regulator of inflammation in disease ${ }^{55}$ has multiple additional binding sites for a variety of cell adhesion receptors. ${ }^{8}$ First, let us consider its RGD motifs only: a single Fgn molecule has a total number of four RGDs $(2 \times 2$ per homodimer, among which the role of two RGDs in cell adhesion remains mostly unclear). ${ }^{53}$ From these data ensues that the surface density of RGD motifs was $340 / 77.1 \cdot 3.1 / 4 \approx 3.4$ times larger in the PLLg-PEG-RGD-layer than in the Fgn-layer. Nonetheless, Fgn was more efficient in promoting cell spreading than PLL$g$-PEG-RGD. Therefore, in Fgn not the RGD motifs, but other binding sites ${ }^{8}$ played the decisive role in determining cell adherence. For example, $\alpha_{\mathrm{M}} \beta_{2}$, the key Fgn-binding integrin expressed by monocytes, MDCs, and MDMs, recognizes numerous sequences on Fgn. ${ }^{56,57}$ Among these, the so-called P2 sequence (amino acids $\gamma 373-395$ ) is probably the most important, ${ }^{53}$ but various sequences can form a complex $\alpha_{\mathrm{M}} \beta_{2}$-binding site with an activity depending on the availability and exposure of its composing elements. ${ }^{57}$ $\alpha_{X} \beta_{2}$, the second most important Fgn-receptor of leukocytes, has a main binding site at the N-terminal domain of the Fgn $\alpha$ chain, and it also binds the P2 sequence. ${ }^{53}$

In summary, although the surface density of RGD motifs was lower on Fgn- than on PLL-g-PEG-RGD-coated surfaces, it is not surprising that cell adhesion was stronger on the former than on the latter. First, the RGD-motifs in Fgn are presumably in a more favorable conformation for binding than in PLL- $g$-PEG-RGD. ${ }^{45}$ Second, Fgn has numerous binding sites other than RGD motifs, and thus potentially much more adhesion receptors are involved in mediating cell adhesion and spreading on Fgn than on PLL-g-PEGRGD. 


\section{Results obtained with three complementary techniques support the biosensor data, but are less information-rich}

Beside the dynamic cell adhesion (DCA) assay, cell adherence 30-60 min after cell seeding was tested with three additional complementary techniques, i.e., with (1) a classical, static adherence assay, (2) a flow-chamber based assay, and (3) an automated micropipette-based assay [Figs. 1(b)-1(d)]. They offer specific advantages. The static adherence assay (see Secs. II G-II I) is the easiest and fastest to carry out, but fluorescent labeling may interfere with cell behavior and the manual washing step limits the precision of the measurement. With the flow-chamber (see Sec. IIH), leukocyte adherence can be tested in a well-defined shear flow, with which the shear flow in blood vessels can be modeled (this is less relevant for MDCs and MDMs, since they typically reside in the tissues). The automated micropipette, a powerful cell sorting technique (see Sec. II I), can probe the force necessary to detach individual cells from the surface. Note, however, that the temporal window in which the flow chamber and automated micropipette techniques can test cell adherence is limited, as very strongly attached cells cannot be removed without damaging the cells (e.g., the plasma membrane is torn before breaking the bonds between the adhesion receptors and their ligands ${ }^{58}$ ). Moreover, a common caveat associated with all three methods is that they yield only end-point data.

All three techniques yielded very similar results regarding the relative adherence of the different cell types on Fgn (Fig. 3), although assay conditions could not be adjusted to be exactly the same (see Secs. IIE and II G-III). Most importantly, cells were preincubated for $1 \mathrm{~h}$ before the classical endpoint measurement, while preincubation time was only $30 \mathrm{~min}$ in the flow chamber and micropipette assays (due to the limited temporal window of these techniques). Still, prominent differences were observed in the adherence of different cell types both in the flow chamber and the automated micropipette assays (Fig. 3). The column plot summarizing the biosensor results in Fig. 2 compare the maximum of the biosensor signals for each experimental condition. In accordance with biosensor data, the results obtained with the complementary techniques suggest that cell types could be ordered by increasing adherence as follows: monocytes, MDMs, and MDCs. In general, data gained with complementary techniques showed larger differences among the adherence of the three cell types as the biosensor data did. Note, however, that in addition to the differences in measuring methods, some details of the assay conditions also differed (e.g., contrasting the protocol followed with complementary techniques, the assay medium did not contain serum, and cells were not preincubated at $37^{\circ} \mathrm{C}$ in the biosensor experiments), which could account for such discrepancies in results. Taking these into consideration, we judge that biosensor data and data gained with the other three techniques are in nice agreement with each other, demonstrating the assay-independent robustness of the observed variances in the adherence of monocytes, MDMs, and MDCs.
Importantly, none of the techniques but the Epic BT enabled the kinetics of cell adhesion and spreading to be monitored and, thus, the adhesive behavior to be impeccably characterized. This proved to be of fundamental advantage, as the investigated immune cells often produced unexpected, nontrivial adhesion kinetic profiles (not symmetrical sigmoid curves with a stable plateau at equilibrium). The biosensor signal started to gradually decrease after $\sim 60 \mathrm{~min}$ of cell seeding (Fig. 2), which suggests that multiple cellular activities may act in concert, and the measured cell adherence and spreading is a net result of their partially opposing effects.

\section{CONCLUSIONS}

The adhesion of monocytes, MFs, and DCs to other cell or to the extracellular matrix plays cardinal roles in immune function, health, and disease. These three cell types all have different main functions that are, to a considerable degree, determined by the differences in adhesive behaviors. However, the adhesion and spreading of monocyes, MDMs, and MDCs has not hitherto been compared in a comprehensive way, still less with an insight to the inherent dynamics of the process.

In order to evaluate differences between the adherence of primary human monocytes, MDMs, and MDCs, we enrolled four different techniques in this study. The classical fluorescence reader-based adherence assay, the flow chamber technique, and the automated micropipette all have their respective advantages, but as a drawback, they yield only end-point data and, thus, can only imperfectly characterize the inherently dynamic process of cell adhesion. In contrast, the Epic BT evanescent-field-based label-free optical biosensor enabled the high-temporal-resolution monitoring of cell adhesion and spreading. Kinetic monitoring revealed that these immune cells, especially monocytes and MDCs, exhibit a complex, unexpected adhesive behavior. The biosensor signal reached its maximum $1 \mathrm{~h}$ after cell seeding, and then it started to gradually decrease. Such a behavior has not been observed previously with other cells types. Nontrivial adhesion and spreading kinetics suggests that multiple cellular activities may act in concert, which have partially opposing effects on total cell adherence and spreading. Indeed, podosomes (multifunctional adhesive and invasive structures particularly prominent in the monocytic lineage) are deeply engaged in both cell-substrate adhesion and matrix degradation and, thus, may be partially responsible for the nonmonotonic biosensor signals. The decreasing signals, observed more prominently in the case of monocytes and MDCs, might also reflect that these are highly migratory cell types.

Exploiting the high-throughput of the biosensor, we could measure the adhesion and spreading of monocytes, MDMs, and MDCs on substrata coated with different molecules. Fibrinogen, an important molecular modulator of immune cell behavior, and PLL-g-PEG-RGD, a simple molecular construct which can specifically induce cell adhesion while blocking nonspecific adsorption, were used as models to 
No force/flow

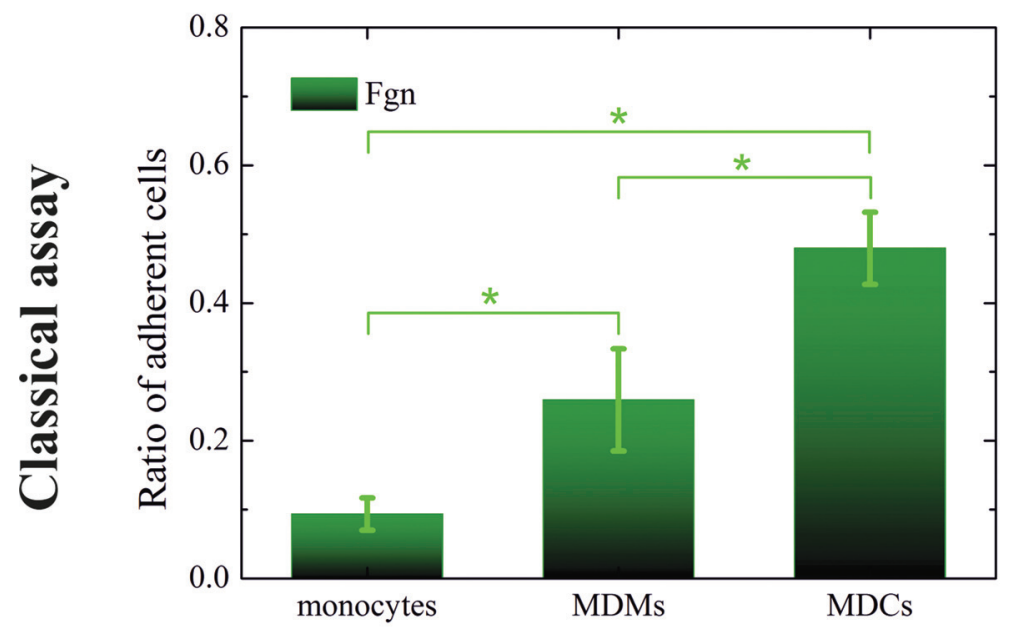

\section{Application of force/flow}
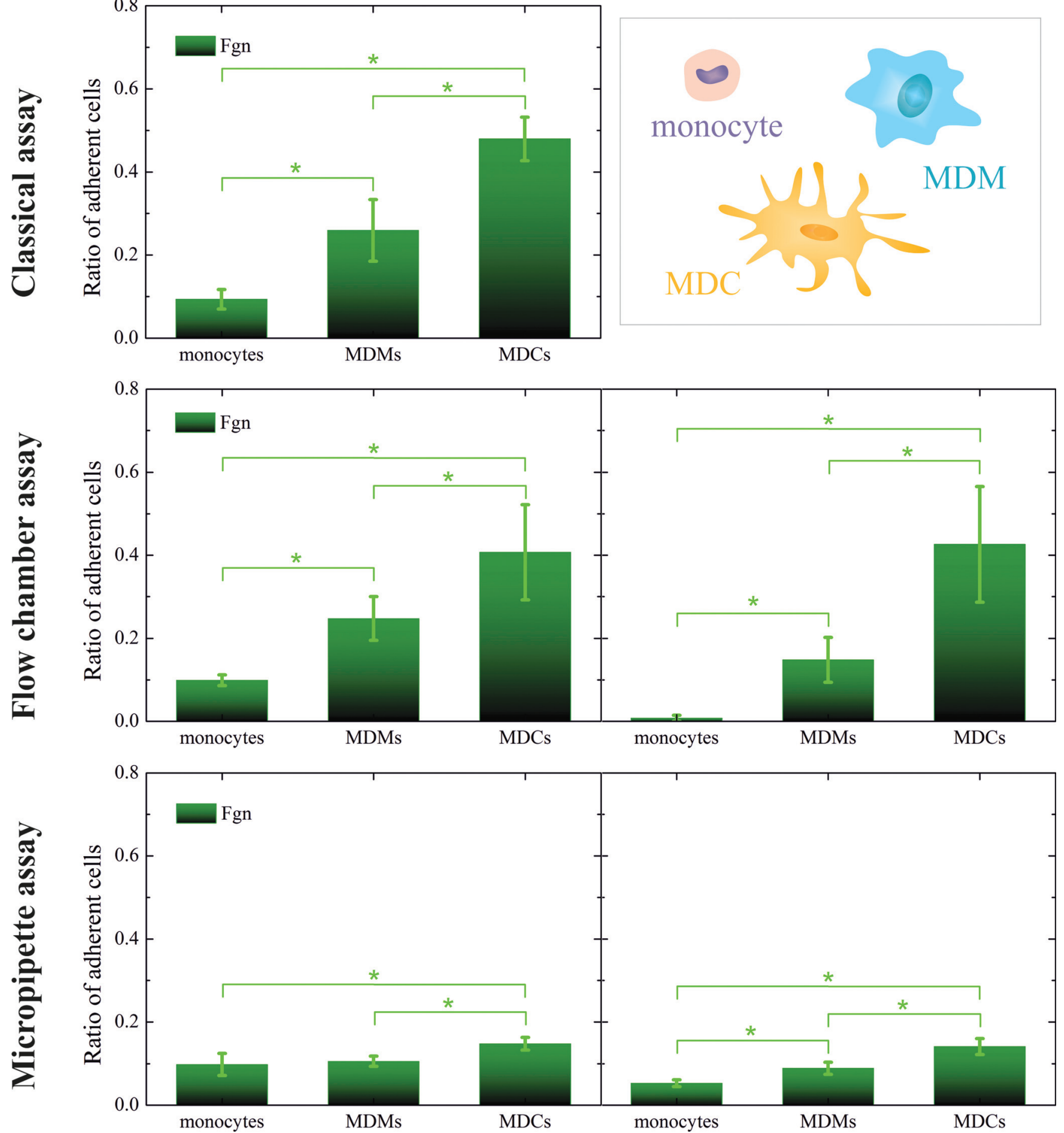

FIG. 3. Adherence of monocytes, MDMs, and MDCs, as was determined with three different techniques complementary to the DCA assays. Top left panel: ratio of adherent cells on Fgn-coated surfaces, as was determined in the classical end-point adhesion assay (see Sec. II G). Cells were preincubated $\left(37^{\circ} \mathrm{C}, \mathrm{CO}_{2}\right)$ in RPMI-10\% FCS for 60 min prior to measurements. Top right panel: schematic illustrating the typical spread morphology of the three investigated cell types. Middle panels: ratio of adherent cells on Fgn-coated surfaces, as was determined in the flow chamber assay (see Sec. II H). Cells were preincubated $\left(37^{\circ} \mathrm{C}, \mathrm{CO}_{2}\right)$ in RPMI-10\% FCS for 30 min prior to experiments. Left and right subpanels show the ratio of adherent cells at shear stresses $\tau=0 \mathrm{~Pa}$ and $\tau=128 \mathrm{~Pa}$, respectively. Bottom panels: cell adherence on Fgn-coated surfaces, as was determined in the automated micropipette assays (see Sec. II I). Cells were preincubated $\left(37^{\circ} \mathrm{C}, \mathrm{CO}_{2}\right)$ in RPMI-10\% FCS for 30 min prior to experiments. Left subpanel shows the ratio of adherent cells after gentle washing, as was determined based on microscope images. Right subpanel shows the ratio of adherent cells after the application of lifting force $(F=1.95 \mu \mathrm{N}$ for monocytes, and $F=1.81 \mu \mathrm{N}$ for MDMs and MDCs) with the micropipette. Data in all panels are shown as background-corrected means (PLL-g-PEG surfaces were used as negative control), error bars represent standard deviations.* indicates statistical significance with $p<0.05$ (t-test). 
create cell-adhesive molecular coatings. According to the biosensor results, cells adhered more firmly on Fgn- than on PLL- $g$-PEG-RGD-coated surfaces. To interpret these results, we characterized the prepared molecular layers by further exploiting the potentials of the biosensor. By measuring the adsorption signal induced during physisorption from the coating solutions to the biosensor wells, we could estimate the surface density of molecules and, thus, the number of binding sites potentially presented for the adhesion receptors. Cells adhered and spread more prominently on Fgnthan on PLL-g-PEG-RGD-coated surfaces despite the fact that the latter presented several times more RGD motifs. Thus, Fgn with its various non-RGD binding sites was able to better promote cell adhesion and spreading, than the simple PLL- $g$-PEG-RGD.

Furthermore, the results obtained with the different techniques also demonstrate that there are significant differences between the adhesion of the three cell types on Fgn. Monocytes were found to be the less adhesive than MDMs and MDCs. Furthermore, MDCs adhered stronger than MDMs; this is in accordance with the fact that MDCs have larger expression levels of $\beta_{2}$ integrins than MDMs. ${ }^{22}$ Hence, it seems that the expression levels of $\beta_{2}$ integrins fundamentally influence the adhesion capacity of these immune cells.

The optical biosensor we used is a robust, yet simpleto-use instrument. It may become a general tool for the investigation of the interaction between adherent immune cells and ECM proteins (see Introduction, for examples).

Meanwhile, several companies and academic research labs continue to invest in further developments, and hence, the spectrum of commercially available label-free optical biosensors is expected to widen in the forthcoming years. An instrument capable of resolving dynamic mass redistributions at the subcellular level ${ }^{59,60}$ would enable the behavior of individual cells to be monitored and thus cell-to-cell variations in a seemingly homogeneous population to be studied. A biosensor combining high-throughput detection with multimode waveguides could be used for the depth profiling of cell refractive index variations, ${ }^{39}$ so the direction of dynamic mass redistributions inside cells could be determined. Highthroughput label-free biosensors equipped with flow-through microfluidics $^{61,62}$ would enable the real-time monitoring of cellular mechanotransduction and could make many cellular assays more biologically relevant by enabling (1) a more controlled stimulation of cells with different substances, and (2) cell behavior to be studied under flow, so the in vivo conditions of red blood cells, various immune cells, and tumor cells circulating in the blood stream could be better approximated. Prompting a more widespread recognition and exploitation of label-free optical biosensors, handheld ${ }^{63}$ and smartphonebased $^{64}$ instruments for point-of-care diagnostics are also at our doorstep, which will surely find cellular applications.

\section{ACKNOWLEDGMENTS}

The present work was supported by the "Lendület" ("Momentum") Program of the Hungarian Academy of
Sciences the ERC_HU grant from NKFIH, and the K104838 OTKA grant. The support from the Bolyai Scholarship to B.S. and MedInProt of the Hung. Acad. Sci. to N.S. and B.S. are gratefully acknowledged. N.S., Z.B., A.E., B.S., and R.H. conceived and designed the experiments; N.O., R.U.S., S.L., and N.S. performed the experiments; S.L. and N.S. isolated monocytes from human blood, differentiated monocytes into dendritic cells and macrophages, labeled cells; N.S., Z.B., E.A., and R.H. supplied reagents/materials; N.O., R.U.S., S.L., N.S., Z.B., A.E., B.S., and R.H. took part in consultations; and N.O. wrote the manuscript and prepared the figures. All authors reviewed and copy edited the manuscript. B.S. is a founder of CellSorter, the startup company that developed the automated micropipette which the authors used in some of the described experiments.

${ }^{1}$ D. C. Dale, L. Boxer, and W. C. Liles, Blood 112, 935 (2008).

${ }^{2}$ F. Geissmann, M. G. Manz, S. Jung, M. H. Sieweke, M. Merad, and K. Ley, Science 327, 656 (2010).

${ }^{3}$ P. D. Yurchenco, Cold Spring Harbor Perspect. Biol. 3, 1 (2011).

${ }^{4}$ L. Sorokin, Nat. Rev. Immunol. 10, 712 (2010).

${ }^{5}$ E. Korpos, C. Wu, J. Song, R. Hallmann, and L. Sorokin, Cell Tissue Res. 339, 47 (2010).

${ }^{6}$ G. G. Vaday and O. Lider, J. Leukocyte Biol. 67, 149 (2000).

${ }^{7}$ H. Schor, G. G. Vaday, and O. Lider, Dev. Immunol. 7, 227 (2000).

${ }^{8}$ M. W. Mosesson, J. Thromb. Haemostasis 3, 1894 (2005).

${ }^{9}$ J. Kreuzer, S. Denger, A. Schmidts, L. Jahn, M. Merten, and E. von Hodenberg, J. Mol. Med. 74, 161 (1996).

${ }^{10}$ T. L. Adair-Kirk and R. M. Senior, Int. J. Biochem. Cell Biol. 40, 1101 (2008).

${ }^{11}$ T. A. Springer, Nature 346, 425 (1990).

${ }^{12}$ A. E. Aplin, A. Howe, S. K. Alahari, R. L. Juliano, and C. Hill, Pharmacol. Rev. 50, 198 (1998).

${ }^{13}$ B. Geiger, J. P. Spatz, and A. D. Bershadsky, Nat. Rev. Mol. Cell Biol. 10 21 (2009).

${ }^{14}$ R. O. Hynes, Cell 110, 673 (2002).

${ }^{15}$ K. Ley, C. Laudanna, M. I. Cybulsky, and S. Nourshargh, Nat. Rev. Immunol. 7, 678 (2007)

${ }^{16}$ B. A. Imhof and M. Aurrand-Lions, Nat. Rev. Immunol. 4, 432 (2004).

${ }^{17}$ A. D. Yurochko, D. Y. Liu, D. Eierman, and S. Haskill, Proc. Natl. Acad. Sci. U. S. A. 89, 9034 (1992).

${ }^{18}$ D. G. Stupack, J. Cell Sci. 115, 3729 (2002).

${ }^{19}$ C. Shi and D. I. Simon, Trends Cardiovasc. Med. 16, 146 (2006).

${ }^{20}$ E. S. Harris, T. M. McIntyre, S. M. Prescott, and G. A. Zimmerman, J. Biol. Chem. 275, 23409 (2000).

${ }^{21}$ M. E. Hemler, Annu. Rev. Immunol. 8, 365 (1990).

${ }^{22}$ C. Ammon, S. P. Meyer, L. Schwarzfischer, S. W. Krause, R. Andreesen, and M. Kreutz, Immunology 100, 364 (2000).

${ }^{23}$ J. Prieto, A. Eklund, and M. Patarroyo, Cell. Immunol. 156, 191 (1994).

${ }^{24}$ R. Salánki, C. Hős, N. Orgovan, B. Péter, N. Sándor, Z. Bajtay, A. Erdei, R. Horvath, and B. Szabó, PLoS One 9, e111450 (2014).

${ }^{25}$ R. B. Wesley, X. Meng, D. Godin, and Z. S. Galis, Arterioscler., Thromb., Vasc. Biol. 18, 432 (1998).

${ }^{26}$ L. M. Shaw, J. M. Messier, and A. M. Mercurio, J. Cell Biol. 110, 2167 (1990).

${ }^{27}$ M. Shen and T. A. Horbett, J. Biomed. Mater. Res. 57, 336 (2001).

${ }^{28}$ N. Orgovan, R. Salánki, N. Sándor, Z. Bajtay, A. Erdei, B. Szabó, and R. Horvath, Biosens. Bioelectron. 54, 339 (2014).

${ }^{29}$ W. J. Kao, D. Lee, J. C. Schense, and J. A. Hubbell, J. Biomed. Mater. Res. 55, 79 (2001).

${ }^{30}$ W. J. Kao, J. A. Hubbell, and J. M. Anderson, J. Mater. Sci. Mater. Med. 10, 601 (1999).

${ }^{31}$ E. Potthoff, O. Guillaume-Gentil, D. Ossola, J. Polesel-Maris, S. LeibundGut-Landmann, T. Zambelli, and J. A. Vorholt, PLoS One 7, e52712 (2012).

${ }^{32}$ G. Sagvolden, I. Giaever, E. O. Pettersen, and J. Feder, Proc. Natl. Acad. Sci. U. S. A. 96, 471 (1999). 
${ }^{33}$ S. S. Hur, Y. Zhao, Y.-S. Li, E. Botvinick, and S. Chien, Cell. Mol. Bioeng. 2, 425 (2009).

${ }^{34}$ J. H.-C. Wang and J.-S. Lin, Biomech. Model. Mechanobiol. 6, 361 (2007).

${ }^{35}$ D. C. Worth and M. Parsons, J. Cell Sci. 123, 3629 (2010).

${ }^{36}$ A. W. Peterson, M. Halter, A. Tona, K. Bhadriraju, and A. L. Plant, Cytometry, A 77A, 895 (2010).

${ }^{37}$ V. Yashunsky, V. Lirtsman, M. Golosovsky, D. Davidov, and B. Aroeti, Biophys. J. 99, 4028 (2010).

${ }^{38}$ K. Tiefenthaler and W. Lukosz, J. Opt. Soc. Am. B 6, 209 (1989).

${ }^{39}$ R. Horvath, K. Cottier, H. C. Pedersen, and J. J. Ramsden, Biosens. Bioelectron. 24, 799 (2008).

${ }^{40}$ J. J. Ramsden and R. Horvath, J. Recept. Signal Transduction Res. 29, 211 (2009).

${ }^{41}$ S. M. Shamah and B. T. Cunningham, Analyst 136, 1090 (2011).

${ }^{42}$ D. Patko, B. Gyorgy, A. Nemeth, K. E. Szabó-Taylor, A. Kittel, E. I. Buzas, and R. Horvath, Sens. Actuators, B 188, 697 (2013).

${ }^{43}$ D. Patko, Z. Mártonfalvi, B. Kovacs, F. Vonderviszt, M. Kellermayer, and R. Horvath, Sens. Actuators, B 196, 352 (2014).

${ }^{44}$ Y. Fang, A. M. Ferrie, N. H. Fontaine, J. Mauro, and J. Balakrishnan, Biophys. J. 91, 1925 (2006).

${ }^{45}$ N. Orgovan, B. Peter, S. Bősze, J. J. Ramsden, B. Szabó, and R. Horvath, Sci. Rep. 4, 4034 (2014).

${ }^{46}$ C. Kecse-Nagy, Z. Szittner, K. Papp, Z. Hegyi, P. Rovero, P. Migliorini, V. Lóránd, L. Homolya, and J. Prechl, PLoS One 11, e0156328 (2016).

${ }^{47}$ A. M. Ferrie, O. D. Deichmann, Q. Wu, and Y. Fang, Appl. Phys. Lett. 100, 223701 (2012).

${ }^{48}$ R. Salánki, T. Gerecsei, N. Orgovan, N. Sándor, B. Péter, Z. Bajtay, A. Erdei, R. Horvath, and B. Szabó, Appl. Phys. Lett. 105, 083703 (2014).
${ }^{49}$ A. Aref, R. Horvath, J. McColl, and J. J. Ramsden, J. Biomed. Opt. 14, 010501 (2009).

${ }^{50}$ T. S. Hug, J. E. Prenosil, and M. Morbidelli, Biosens. Bioelectron. 16, 865 (2001).

${ }^{51}$ S. Linder and C. Wiesner, Cell. Mol. Life Sci. 72, 121 (2015).

${ }^{52}$ N. Orgovan, B. Kovacs, E. Farkas, B. Szabó, N. Zaytseva, Y. Fang, and R. Horvath, Appl. Phys. Lett. 104, 083506 (2014).

${ }^{53}$ T. P. Ugarova and V. P. Yakubenko, Ann. New York Acad. Sci. 936, 368 (2001).

${ }^{54}$ J. A. G. Van Strijp, D. G. Russell, E. Tuomanen, J. Brown, and S. D. Wright, J. Immunol. 151, 3324 (1993).

${ }^{55}$ D. Davalos and K. Akassoglou, Semin. Immunopathol. 34, 43 (2012).

${ }^{56}$ V. K. Lishko, N. P. Podolnikova, V. P. Yakubenko, S. Yakovlev, L. Medved, S. P. Yadav, and T. P. Ugarova, J. Biol. Chem. 279, 44897 (2004).

${ }^{57}$ S. Yakovlev, L. Zhang, T. Ugarova, and L. Medved, Biochemistry-US 2, 617 (2005).

${ }^{58}$ A. S. Goldstein and P. A. DiMilla, J. Biomed. Mater. Res. A 67A, 658 (2003).

${ }^{59}$ N. K. Febles, A. M. Ferrie, and Y. Fang, Anal. Chem. 86, 8842 (2014).

${ }^{60}$ L. L. Chan, S. L. Gosangari, K. L. Watkin, and B. T. Cunningham, Sens. Actuators, B 132, 418 (2008).

${ }^{61}$ V. Goral, Q. Wu, H. Sun, and Y. Fang, FEBS Lett. 585, 1054 (2011).

${ }^{62}$ N. Orgovan, D. Patko, C. Hos, S. Kurunczi, B. Szabó, J. J. Ramsden, and R. Horvath, Adv. Colloid Interface Sci. 211, 1 (2014).

${ }^{63}$ S. Jahns et al., Biomed. Opt. Express 6, 3724 (2015).

${ }^{64}$ D. Gallegos, K. Long, H. Yu, P. Clark, Y. Lin, S. George, P. Nath, and B. Cunningham, Lab Chip 12, 2124 (2012). 